\title{
Observation of the Production of Jets of Particles at High Transverse Momentum and Comparison with Inclusive Single-Particle Reactions*
}

C. Bromberg, G. Fox, R. Gomez, J. Pine, S. Stampke, and K. Yung California Institute of Technology, Pasadena, California 91125

and

S. Erhan, E. Lorenz, $\dagger$ M. Medinnis, J. Rohlf, and P. Schlein University of California, Los Angeles, Califormia 90024

and

V. Ashford, H. Haggerty, R. Juhala, Fermi National Accelerator Laboratory, Batavia, Illinois 60510

and

R. Abrams, R. Delzenero, H. Goldberg, S. Margulies, D. McLeod, J. Solomon, and R. Stanek University of Mlinois at Chicago Circle, Chicago, Illinois 60680

and

A. Dzierba and W. Kropac $\S$

Indiana University, Bloomington, Indiana 47401

(Received 11 April 1977)

\begin{abstract}
Data are presented on production by $200-\mathrm{GeV} / c$ hadrons incident on beryllium of both single particles and jets (groups of particles) with high $p_{T}$ (transverse momentum). The experiment was performed in a wide-aperture multiparticle spectrometer at Fermilab. The jet and single-particle cross sections have a similar shape from $p_{T}=3$ to $5 \mathrm{GeV} / \mathrm{c}$ but the jet cross section is over two orders of magnitude larger. The distributions of charged-particle momenta show striking similarities to those observed in lepton-induced processes.
\end{abstract}

Interest in high- $p_{T}$ physics was originally stimulated by the observation of unexpectedly large yields of single particles for $p_{T} \gtrless 3 \mathrm{GeV} / c .^{1}$ The motivation of our experiment is to shed light on the dynamics of these rare processes by studying both the particles produced in conjunction with a single high $-p_{T}$ particle and the structure of events in which high $p_{T}$ is found as the sum of the transverse momenta of several particles. The data presented in this Letter are the result of an initial run at Fermilab and details of the apparatus and additional data will be presented elsewhere. ${ }^{2}$

The spectrometer is shown schematically in Fig. 1. Beam particles with a momentum of 200 $\mathrm{GeV} / c$ struck a beryllium target either 3 or 6 cm thick. Charged particles emerging from the target at lab angles $\lesssim 150 \mathrm{mrad}$ and momenta $\gtrsim 2$ $\mathrm{GeV} / c$ were analyzed by track chambers and the spectrometer magnet which was set to give a transverse momentum kick of $0.38 \mathrm{GeV} / c$. Behind the last track chamber were two calorimeters, each divided into four separate modules.
The modules consisted of a front section comprised of six alternating layers of $0.25 \mathrm{in}$. of scintillator and 0.56 in. of lead, totaling about 15 radiation lengths or about 0.5 nuclear absorption length, and a rear section comprised of fifteen layers of $0.25 \mathrm{in}$. of scintillator and 2 in. of iron, totaling about 4.5 absorption lengths. The lead (electron) sections and iron (hadron) sections were viewed by separate phototubes. In addition, each module was viewed at the top and bottom. Pulse-height analysis gave a measure of the energy and mean vertical position of the interactions.

Event triggers for the experiment were derived from the calorimeter module signals, weighted by their mean lab angle so that they were an approximate measure of transverse momentum. Two types of trigger were used, which we have called single particle and jet. For the former, it was required that the sum of the transverse momenta in the electron and hadron sections of any individual module be greater than a preset trigger bias of $3 \mathrm{GeV} / c$. The jet trigger demanded that 


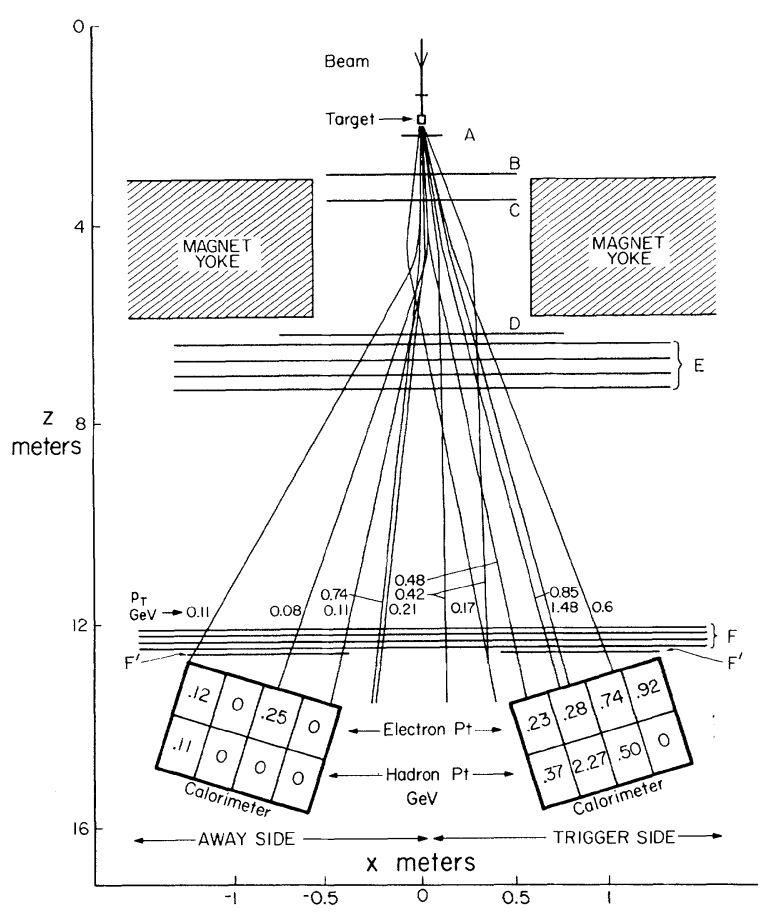

FIG. 1. Plan view of the spectrometer, with tracks from a typical event. $A-D$ and $F^{\prime}$ indicate proportional planes, while $E$ and $F$ are spark chambers

the total transverse momentum of all four modules of either calorimeter be above a preset level: Data with thresholds of 3,4 , and $4.5 \mathrm{GeV} / c$ were used in this analysis. In Fig. 1, the jet-trigger $p_{T}$ is $5.31 \mathrm{GeV} / c$ in the right-hand-side calorimeter.

The mean number of reconstructed tracks which come from the beryllium vertex and pass through the magnet is about ten for jets and one less for single-particle triggers. Furthermore the chambers in front of the magnet measure, on the average, four to five additional charged particles per event which are outside the magnet acceptance. Individual spark-chamber-gap efficiencies are estimated at $90 \%$ up to multiplicities of fifteen and the overall efficiency of track finding, away from the beam direction, is estimated to be (95 $\pm 5) \%$. The number of spurious high-transversemomentum tracks is shown to be small both by comparing calorimeter and track energies and also by the lack of a high-momentum tail in our charged-particle $p_{T}$ spectrum. The momentum resolution of the track chambers is $\Delta p / p^{2}=0.0007$ $(\mathrm{GeV} / c)^{-1}$. For the calorimeters we measure an energy resolution $\Delta E / E$ of $0.33 / \sqrt{E}$ for electron modules and 1.03/ $\sqrt{E}$ for hadron modules (stan-

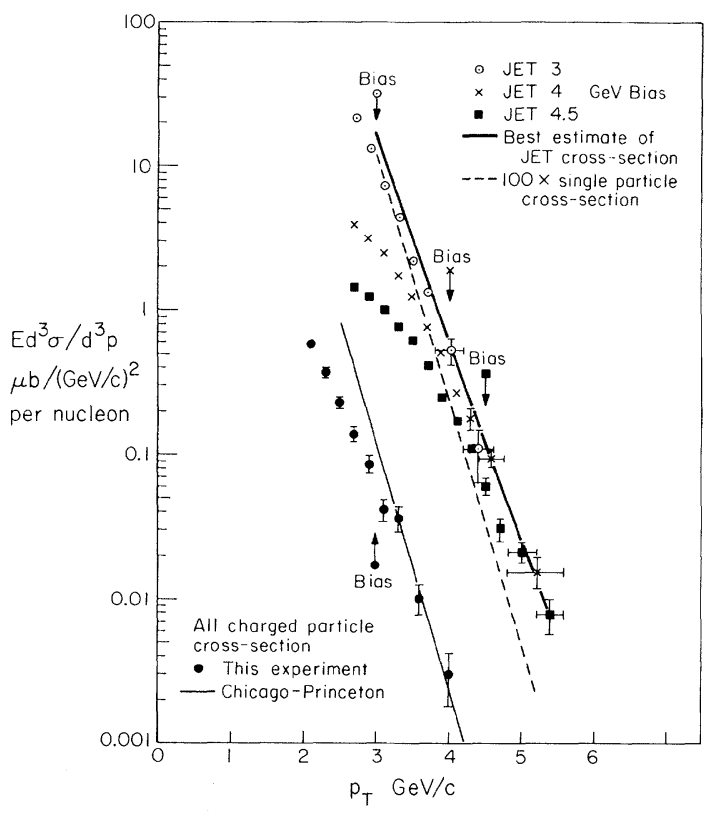

FIG. 2. The single-particle and jet cross sections averaged over the c.m. system rapidty $(y)$ region of 0.1 to 0.44 . The jet definition is all particles into the calorimeter, while the plotted data are an average of equal amounts of $\pi^{-}$and proton beam. The jet cross sections for the separate beam types $\pi^{-}$and $p$ are equal to within $20 \%$ for $4<p_{\perp} \mathrm{JET}^{2}<\mathrm{GeV} / c$. The singleparticle data correspond to a proton beam only and the Chicago-Princeton results (Ref. 3) were taken at a lab angle of $77 \mathrm{mrad}(y \approx 0.25)$ and have been summed over all charged particles for comparison with our data.

dard deviations, $E$ in $\mathrm{GeV}$ ).

The single-particle trigger is straightforward to analyze. Taking events with a single-particle track with $p_{T}>2 \mathrm{GeV} / c$, we find the acceptancecorrected invariant cross section plotted in Fig. 2 using a sample of the events in which the track enters a fiducial region in the middle of the calorimeter. Our data can be directly compared to the more precise measurements of the ChicagoPrinceton $^{3}$ collaboration and there is good agreement above the calorimeter trigger threshold of $3 \mathrm{GeV} / c$.

The novel feature of our experiment is the jet trigger defined above. The contributions to jet $p_{T}$ divide into three categories: (i) charged particles (typical multiplicity three) measured by the track chambers and comprising $57 \%$ of the jet momentum ${ }^{4}$; (ii) photons (presumably from $\pi^{0}, \eta$, etc., decay) measured in the electron calorimeter and making up $30 \%$ of the jet momentum; and (iii) neutral hadrons or apparent neutrals. These latter are deduced by subtracting the charged- 
track energies from the hadron calorimeter signals module by module; the resultant resolution in energy is poor because of the subtraction and modest calorimeter resolution. This third category includes neutrons, $K_{L}{ }^{0}$, and nonvertex tracks $\left(K_{s}{ }^{0}, \Lambda\right.$, and reconstruction losses) and we find that it carries between 6 and $24 \%$ of the jet $p_{T}$ with $13 \%$ as our best estimate. The jet cross section presented in Fig. 2 has been corrected for geometric acceptance but not for the trigger bias of the calorimeter. However, comparison of the three trigger thresholds shown in Fig. 2 allows us to extract an unbiased jet cross section shown as the broad solid line. The uncertainty in the hadronic neutral component, discussed above, leads to a cross-section uncertainty of $\pm 30 \%\left(p_{T}\right.$ $=3 \mathrm{GeV} / \mathrm{c})$ to $\pm 50 \%\left(p_{T}=5 \mathrm{GeV} / c\right)$ not indicated in Fig. 2.

Independent of the uncertainty in the hadronic neutral estimate, the striking feature of Fig. 2 is the very large (over two orders of magnitude) ratio of jet to single-particle cross section. This result had been predicted in models ${ }^{5-7}$ which postulate that large $-p_{T}$ hadrons come from the fragmentation of quarks produced in an elementary hard collision between constituent quarks in the initial hadrons. Note that there are no clear resonance signals in our jet data and the large jet cross section is not simply due to a sum of low-

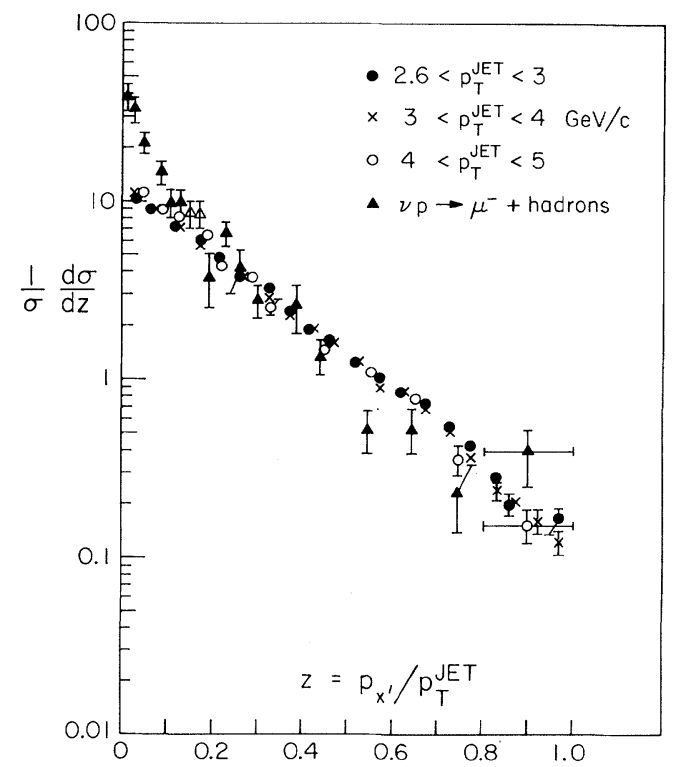

FIG. 3. The distribution in transverse momentum fraction $z$ for three jet $p_{\perp}$ ranges compared with neutrino scattering (Refs. 7 and 8). The comparison fails below $z \sim 0.2$ as the neutrino data have significant contributions from the fragmentation of the target proton. mass resonances.

Motivated by the constituent models, we define the jet plane as that plane formed by the jet momentum vector and the direction of the incident hadrons and plot in Fig. 3 the transverse momentum fraction $z=p_{x^{\prime}} / p_{T}^{\mathrm{JET}}$, where $p_{T}^{\mathrm{JET}}$ is the transverse momentum of the jet and $p_{x}$, is the component of the transverse momentum of individual charged particles in the jet plane. There is a striking similarity between our $z$ distributions and those for lepton-induced processes. ${ }^{7,8}$ Furthermore our data show evidence for the coplanar structure of events suggested by constituent models. For the charged particles (both jet and nonjet members) in these events we find a sharp exponential dependence with a mean of 0.20 $\mathrm{GeV} / c$ for the component of the transverse momentum out of the jet plane. Also the two-dimensional momentum perpendicular to the jet axis is strongly cut off for the jet members. In Ref. 2, we explore fully these points and the relation of our experimental jet definition to theory.

Finally Fig. 4 shows the momentum distribution of charged particles on the away side (cf. Fig. 1). The first striking feature is that these away-side particles balance the transverse momentum of

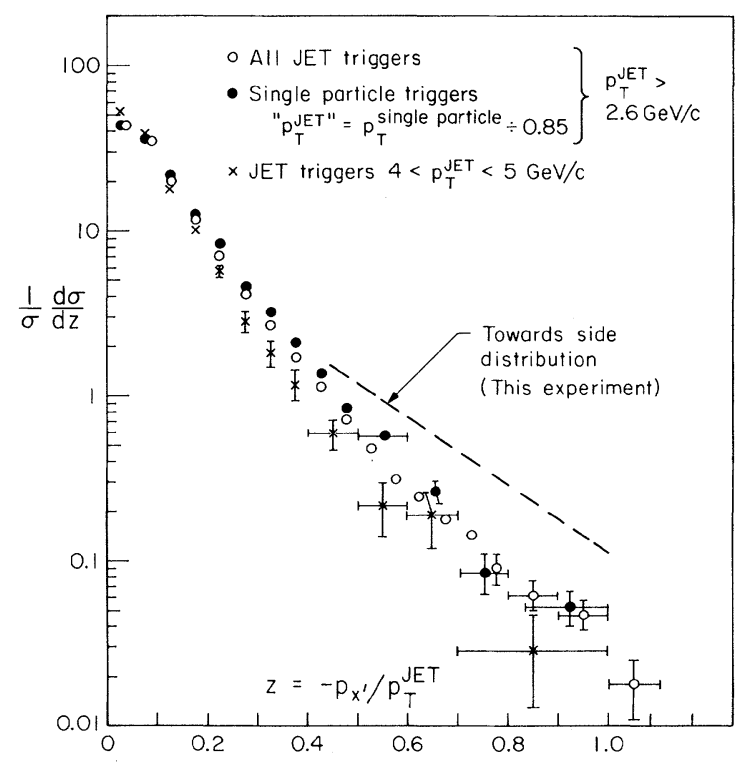

FIG. 4. Distributions in momentum fraction $z$ for particles on the away side. The shifting of scale for single particles is motivated by the quark model (Refs. 7 and 9) which suggests that single particles carry from 80 to $90 \%$ of the momentum of the high- $p_{\perp}$ quark (jet). The data are integrated over the whole away side and include a correction for spectrometer acceptance which is valid for the higher-momentum particles, $z \gtrsim 0.25$. 
single-particle and jet triggers in a similar way. This is expected in the quark picture ${ }^{7,9}$ but seems difficult to understand in the constituent interchange model. ${ }^{10}$ Again, the away- and trigger(towards) side $z$ distributions have similar shapes but differ by a factor of $\sim 2.5$ in magnitude at large $z$. In constituent models, this difference is a direct reflection of the transverse momentum of quarks inside hadrons. ${ }^{9}$

Our data give strong support to the idea that the same constituents (quarks presumably) are probed by both high- $p_{T}$ hadron and lepton collisions. We are currently continuing our analysis on the completed experiment which has 10 times the data presented here, taken with a liquid-hydrogen target and incident proton and meson beams.

We are grateful for the assistance given to us by the staffs of the Accelerator Division, the Meson Department, and the Research Services Department at Fermilab.

*Work supported in part by the U. S. Energy Research and Development Administration under Contracts No. $\mathrm{E}(11-1)-68$ (Caltech) and No. E(11-1)-2009 (Indiana); and by the National Science Foundation under Grants No. PHY-76-80660 (U.C.L.A.) and No. MPS-73-04640 (Illinois).

$\dagger$ Permanent address: Max-Planck-Institut für Physik und Astrophysik, Munich, Germany.

$\$$ Presently at Oak Ridge National Laboratory, Oak
Ridge, Tenn. 37830 .

§Presently at California Institute of Technology, Pasadena, Calif. 91125.

${ }^{1} \mathrm{P}$. Darriulat, in Proceedings of the Eighteenth International Conference on High Energy Physics, Tbilisi,

U. S. S. R., 1976, edited by N. N. Bogolubov et al. (The Joint Institute for Nuclear Research, Dubna, U.S.S.R., 1977).

${ }^{2}$ C. Bromberg et al ., "Production of Jets and SingleParticles at High $p_{T}$ in 200-GeV Hadron Beryllium Collisions" (to be published).

${ }^{3} \mathrm{~J}$. W. Cronin et al., Phys. Rev. D 11, 3105 (1975)。 Figure 2 shows more recent data [D. Antreasyan et al., Phys. Rev. Lett. 38, 115 (1977), and private communication of new results with a Be targetl.

${ }^{4}$ The percentage of jet $p_{T}$ carried by the three components varies slightly with the jet $p_{T}$ and more significantly with the hadronic neutral estimate used. The quoted percentages correspond to the cut $4<p_{T}{ }^{\mathrm{JET}}<5$ $\mathrm{GeV} / c$ and a $13 \%$ hadronic neutral contribution.

${ }^{5}$ S. M. Berman, J. D. Bjorken, and J. B. Kogut, Phys. Rev。D 4, 3388 (1971).

${ }^{6}$ S. D. Ellis and M. B. Kislinger, Phys. Rev. D $\underline{9}$, 2027 (1974).

${ }^{7}$ R. D. Field and R. P。Feynman, Phys. Rev. D $\underline{15}$, 2590 (1977).

${ }^{8} \mathrm{~J}$. C. Vander Velde, "Neutrino-Proton Interactions in the 15-Foot Bubble Chamber and Properties of Hadron Jets" (to be published).

${ }^{9}$ G. C. Fox, in BNL Report No. BNL-50598, edited by H. Gordon and R. F. Peierls, Proceedings of the American Physical Society Meeting, Division of Particles and Fields, Upton, New York, 1976 (unpublished).

${ }^{10}$ D. Sivers, S. Brodsky, and R. Blankenbecler, Phys. Rep. 23C, 1 (1976).

\title{
Measurement of the Proton Structure Function from Muon Scattering*
}

H. L. Anderson, V. K. Bharadwaj, N. E. Booth, R。 M. Fine, $\dagger$ W. R. Francis, $\ddagger$ B. A. Gordon, R. H. Heisterberg, § R. G. Hicks, T. B. W. Kirk, " G. I. Kirkbride, १

W. A. Loomis, H. S. Matis, L. W. Mo, § L. C. Myrianthopoulos, F. M. Pipkin, S. H. Pordes, ** T. W. Quirk, W. D. Shambroom, A. Skuja, $\dagger \dagger$ M. A. Staton, W. S. C. Williams, L. J. Verhey, Richard Wilson, and S. C. Wright

Enrico Fermi Institute, The University of Chicago, Chicago, Mlinois 60637, and High Energy Physics Laboratory and Department of Physics, Harvard University, Cambridge, Massachusetts 02138, and Department of Physics,

The University of Mlinois at Urbana-Champaign, Urbana, Illinois 61801, and Department of Nuclear Physics, The University of Oxford, Oxford OX1 3RH, England

(Received 25 April 1977)

\begin{abstract}
Results on the proton structure function, $\nu W_{2}$ are presented for $0.3<q^{2}<50 \mathrm{GeV}^{2}$ and 5 $<\nu<130 \mathrm{GeV}$. They are compared to earlier data and displayed to demonstrate violations of scaling. Values are reported for the energy-momentum sum rule and for $R \equiv \sigma_{L} / \sigma_{T}$ over a limited kinematic region.
\end{abstract}

In this Letter we report values of the structure function $\nu W_{2}$ of the proton obtained by measuring the inelastic scattering of muons from hydrogen at the Fermi National Accelerator Laboratory. The data were taken with the muon-scattering facility constructed by this group. 